\title{
Leucine supplementation improves adiponectin and total cholesterol concentrations despite the lack of changes in adiposity or glucose homeostasis in rats previously exposed to a high-fat diet
}

Francisco L Torres-Leal ${ }^{1 *}$, Miriam H Fonseca-Alaniz² , Gabriela FR Teodoro ${ }^{1}$, Mariana D de Capitani ${ }^{1}$, Daiana Vianna ${ }^{1}$, Lucas C Pantaleão ${ }^{1}$, Emidio M Matos-Neto ${ }^{1}$, Marcelo M Rogero ${ }^{3}$, Jose Donato $\mathrm{Jr}^{4}$ and Julio Tirapegui ${ }^{1}$

\begin{abstract}
Background: Studies suggest that leucine supplementation (LS) has a therapeutic potential to prevent obesity and to promote glucose homeostasis. Furthermore, regular physical exercise is a widely accepted strategy for body weight maintenance and also for the prevention of obesity. The aim of this study was to determine the effect of chronic LS alone or combined with endurance training (ET) as potential approaches for reversing the insulin resistance and obesity induced by a high-fat diet (HFD) in rats.

Methods: Forty-seven rats were randomly divided into two groups. Animals were fed a control diet-low fat $(n=$ 10) or HFD ( $n=37)$. After 15 weeks on HFD, all rats received the control diet-low fat and were randomly divided according to treatment: reference (REF), LS, ET, and LS+ET ( $n=7-8$ rats per group). After 6 weeks of treatment, the animals were sacrificed and body composition, fat cell volume, and serum concentrations of total cholesterol, HDLcholesterol, triacylglycerol, glucose, adiponectin, leptin and tumor necrosis factor-alpha (TNF- $\alpha$ ) were analyzed.

Results: At the end of the sixth week of treatment, there was no significant difference in body weight between the REF, LS, ET and LS+ET groups. However, ET increased lean body mass in rats $(P=0.019)$. In addition, ET was more effective than LS in reducing adiposity $(P=0.019)$, serum insulin $(P=0.022)$ and TNF- $\alpha(P=0.044)$.

Conversely, LS increased serum adiponectin $(P=0.021)$ levels and reduced serum total cholesterol concentration $(P$ $=0.042$ ).
\end{abstract}

Conclusions: The results showed that LS had no beneficial effects on insulin sensitivity or adiposity in previously obese rats. On the other hand, LS was effective in increasing adiponectin levels and in reducing total cholesterol concentration.

Keywords: Leucine, Adipose tissue, Endurance training, Body composition, Obesity, Adiponectin

\section{Background}

Obesity is associated with a number of health problems that are often summarized as the metabolic syndrome, and its etiology may be associated with the consumption of high energy-dense foods [1]. Excessive intake of dietary fat promotes adipocyte hypertrophy, altering their normal endocrine function to an inflammatory

\footnotetext{
* Correspondence: torresleal@usp.br

'Department of Food Science and Experimental Nutrition, Faculty of

Pharmaceutical Sciences, University of São Paulo, São Paulo, Brazil

Full list of author information is available at the end of the article
}

pathologic condition that increases the secretion of tumor necrosis factor-alpha (TNF- $\alpha$ ) and interleukin- 6 (IL-6), among other proinflammatory cytokines, and concomitantly reducing adiponectin secretion $[2,3]$.

Previous studies have demonstrated that dietary leucine regulates body weight and glucose homeostasis [410]. Within this context, several studies support the hypothesis that leucine plays an important role in the regulation of metabolism and energy balance by directly affecting peripheral tissues, such as white adipose tissue, liver, and muscle. For example, leucine has been shown 
to increase the secretion of leptin [11] and adiponectin $[12,13]$ from adipocytes.

Another important and widely accepted strategy for the maintenance of body weight and prevention of obesity is regular physical exercise $[14,15]$. The beneficial effects of physical exercise on weight loss are attributed to body fat reduction, maintenance of lean body mass and improvement of metabolic condition, e.g., increasing glucose uptake, especially in skeletal muscle $[14,15]$. Moreover, chronic physical exercise has several effects secondary to body fat loss, including a reduction in the synthesis and secretion of proinflammatory adipokines $[16,17]$ and an increase in circulating levels of adiponectin, the main anti-inflammatory molecule secreted by adipocytes $[18,19]$. Physical exercise increases energy expenditure, exerting a greater effect during a state of negative energy balance, especially when combined with a reduction in the consumption of high energy-dense foods [20].

Within this context, in which the most recommended lifestyle changes for the treatment of obesity and diabetes include an adequate diet and regular physical exercise, leucine supplementation (LS) and physical exercise may affect the maintenance of body weight and, possibly, the metabolic status. Therefore, the present study was designed to evaluate the potential therapeutic effect of these two factors on the treatment of obesity. For this purpose, adult rats previously treated for 15 weeks with a high-fat diet (HFD) received chronic LS alone or combined with endurance training (ET). The effects of 6week LS on body composition, food intake, adipocyte volume, and serum levels of several pro- and antiinflammatory metabolic markers were assessed.

\section{Methods and Procedures}

Animals

Male Sprague-Dawley rats (approximately 120 days old) were obtained from the Animal Laboratory of the Faculty of Pharmaceutical Sciences at the University of São Paulo and were kept with food and water ad libitum. The rats were maintained in a room at an ambient temperature of $22 \pm 2^{\circ} \mathrm{C}$ and a relative humidity of $55 \pm$ $10 \%$ under a 12 -h light/12-h dark cycle. All animal procedures were approved by the Ethics Committee for animal experimentation of the Faculty of Pharmaceutical Sciences, University of São Paulo, according to the guidelines of the Brazilian College on Animal Experimentation.

\section{Diet and experimental design}

The experimental diets were prepared according to the recommendations of the American Institute of Nutrition for adult rats and are shown in Table 1[21]. After weight distribution, rats were fed the control diet $(\mathrm{CD}$,
Table 1 Diet composition ${ }^{1}$

\begin{tabular}{|c|c|c|c|}
\hline & Control diet & High-fat diet & + Leucine diet \\
\hline & & $\mathrm{g} / \mathrm{kg}$ diet & \\
\hline Cornstarch & 620.6 & 282.6 & 570.6 \\
\hline Casein & 140.0 & 140.0 & 140.0 \\
\hline Sucrose & 100.0 & 100.0 & 100.0 \\
\hline Soybean oil & 40.0 & 40.0 & 40.0 \\
\hline Lard & - & 338.0 & - \\
\hline Cellulose & 50.0 & 50.0 & 50.0 \\
\hline AIN-93M mineral mixture & 35.0 & 35.0 & 35.0 \\
\hline AIN-93M vitamin mixture & 10.0 & 10.0 & 10.0 \\
\hline Choline bitartrate & 2.5 & 2.5 & 2.5 \\
\hline L-Cystine & 1.8 & 1.8 & 1.8 \\
\hline tert-Butylhydroquinone & 0.008 & 0.008 & 0.008 \\
\hline L-Leucine & - & - & 50.0 \\
\hline Total (g) & 1.000 & 1.000 & 1.000 \\
\hline
\end{tabular}

${ }^{1}$ Based on AIN-93M (21).

$n=10)$ or HFD $(n=37)$ for 15 weeks. At the end of the first 15 weeks, 10 animals in the $C D$ group and 7 animals in the HFD group were sacrificed in order to evaluate their metabolic status. After 15 weeks on HFD, the obese animals were divided into four subgroups of treatment for 6 weeks: i) reference (REF) group (REF, $n$ $=7$ ) receiving the $\mathrm{CD}$; ii) LS group (LS, $n=8$ ) receiving diet supplemented with 5\% L-leucine (Ajinomoto Interamericana Indústria e Comércio Ltda, São Paulo, Brazil); iii) endurance training group (ET, $n=8)$ receiving the $\mathrm{CD}$, and iv) LS plus ET group receiving diet supplemented with $5 \%$ L-leucine (LS+ET, $n=7)$. Other studies used this leucine dose and no toxic effect was reported $[22,23]$.

Body weight and food intake were assessed weekly and final body weight was recorded immediately before euthanasia. At the end of 21 weeks, rats were fasted for 12 hours, and 72 hours after the last exercise session, the animals were anesthetized with rodent cocktail [xylazine hydrochloride $(20 \mathrm{mg} / \mathrm{mL})$, ketamine hydrochloride (100 $\mathrm{mg} / \mathrm{mL})$, acepromazine $(20 \mathrm{mg} / \mathrm{mL})$, and distilled water $(4.5 / 4.5 / 1.8 / 7.2, \mathrm{v} / \mathrm{v})]$. Trunk blood samples were collected after decapitation and centrifuged and serum was stored in a freezer at $-80^{\circ} \mathrm{C}$. The gastrointestinal tract was completely emptied and washed with saline. Subcutaneous (SC), epididymal (EP), and retroperitoneal (RP) white adipose fat pads were totally excised, weighed, and processed for adipocyte isolation. Body composition was determined by chemical analysis of the carcass as previously described by Donato et al. [5].

\section{Endurance training}

The exercise program consisted of continuous swimming in individual tanks filled with water and maintained at $28-32^{\circ} \mathrm{C}$. Animals were trained for 6 weeks 
during 60-min daily sessions, five times a week. All rats swam with a load of $5 \%$ body weight, which was attached to their tail. This training protocol is already standardized in our laboratory $[24,25]$.

\section{Adipocyte isolation}

Adipocytes were isolated with collagenase as previously described [26]. The isolated adipocytes $\left(\sim 7\right.$ to $8 \times 10^{5}$ cells $/ \mathrm{mL}$ ) were resuspended in EHB buffer (Earle's salts, $20 \mathrm{mM}$ HEPES, 1\% bovine serum albumin, $2 \mathrm{mM}$ sodium pyruvate, and $4.8 \mathrm{mM}$ sodium bicarbonate), $\mathrm{pH}$ 7.4 at $37^{\circ} \mathrm{C}$. For morphometric analysis, aliquots of the cell suspension were used to measure the transverse diameter of 100 isolated adipocytes with a light microscope equipped with a micrometer. Assuming that the isolated adipocyte is spherical, this value was used to calculate the volume and average cell surface area according to the formulas proposed by Fine \& Digirolamo [27]. The number of adipocytes was determined by the ratio of total tissue weight (pg) to average adipocyte mass (pg).

\section{Serum analysis}

Serum levels of total cholesterol, high-density lipoprotein (HDL)-cholesterol, triacylglycerol (TG), and glucose were determined by enzymatic methods using commercial kits (Labtest Diagnóstica kit, Glucose PAP Liquiform, São Paulo, Brazil). Serum leptin and insulin concentrations were quantified using radioimmunoassay kits (Linco Research, Inc., St Charles, MO, USA). Serum adiponectin level was determined with an enzyme-linked immunosorbent assay (ELISA) kit (Linco Research, Inc.). Serum TNF- $\alpha$ d was quantified using the LINCOplex assay (Linco Research, Inc.). The blood concentration of amino acids was determined by the methods of White et al. [28] and Hagen et al. [29]. The homeostasis model assessment (HOMA) index was calculated as an indicator of insulin resistance: HOMA = (fasting plasma insulin concentration $(\mathrm{ng} / \mathrm{mL})) \times($ fasting plasma glucose $(\mathrm{mmol} / \mathrm{L})) / 22.5$

The ratio adiponectin/fat pad weight ratio was calculated by dividing the basal adiponectin for fat pad weight.

\section{Statistical analysis}

Data are expressed as mean \pm SEM. The unpaired twotailed Student $t$ test was used to evaluate obesity status. The effect of LS and ET was analyzed by factorial ANOVA $\left(2^{2}\right)$ followed by Tukey's honestly significant difference test. ANOVA for repeated measures was used for the comparison of body weight, followed by Dunnett's test when comparing times. Differences were considered to be significant when $P \leq 0.05$. Calculations were performed and graphs were drawn using the Statistica version 7.1 program (StatSoft).

\section{Results}

Characterization of obesity model, hyperglycemia and hyperinsulinemia

Administration of the HFD for 15 weeks induced a significant increase in total adiposity $(P=0.024)$, including increases in SC $(P=0.045), \mathrm{EP}(P=0.004)$ and RP $(P$ $<0.001)$ fat pads and hypertrophy of SC $(P=0.041)$ and EP $(P=0.014)$ adipocytes when compared to the CD group (Table 2).

We also observed a significant increase in blood glucose $(12 \%)(P=0.035)$, insulin $(86 \%)(P=0.022)$, TNF$\alpha(28 \%)(P=0.010)$, and leptin $(130 \%)(P<0.001)$ levels in the HFD group compared to the CD group. On other hand, serum adiponectin concentration was significantly $(P=0.006)$ reduced $(-27 \%)$ in the HFD group (Table 2$)$. Taken together, these results indicate that the HFD caused obesity and insulin resistance and altered the blood levels of major adipokines in rats.

\section{LS and ET in the treatment of diet-induced obesity}

After induction of obesity with HFD, the groups of rats started the treatments with a similar initial body weight. No significant differences in daily energy intake were observed between groups: REF (90.26 $\pm 9.37 \mathrm{kcal} /$ day); LS $(97.75 \pm 7.51 \mathrm{kcal} /$ day $) ; \mathrm{ET}(98.75 \pm 10.44 \mathrm{kcal} /$ day $)$; LS+ET $(82.26 \pm 11.19 \mathrm{kcal} /$ day $)$; diet $(P=0.655)$; training $(P=0.728)$, and interaction $(P=0.252)$. During the fifth week of the experiment, physical training significantly $(P=0.045)$ reduced body weight. However, at the end of the sixth week of treatment, there was no significant difference $(P>0.05)$ in body weight between the REF, LS, ET and LS+ET groups (Figure 1A). In addition, leucine exerted a transient effect on the reduction of body weight in the LS group in the second and third week $(P=0.002)$. In parallel, LS+ET caused

Table 2 Obesity state and metabolic parameters in rats after 15 weeks of dietary treatment

\begin{tabular}{llll}
\hline & Control diet & High-fat diet & $P$ \\
\hline Body fat, $\%$ & $14.46 \pm 1.16$ & $20.94 \pm 2.39^{*}$ & 0.024 \\
SC fat pad, $g / 100 \mathrm{~g}$ & $2.77 \pm 0.17$ & $4.09 \pm 0.69^{*}$ & 0.045 \\
EP fat pad, $\mathrm{g} / 100 \mathrm{~g}$ & $1.98 \pm 0,14$ & $2.72 \pm 0.17^{*}$ & 0.004 \\
RP fat pad, $\mathrm{g} / 100 \mathrm{~g}$ & $1.40 \pm 0.09$ & $2.29 \pm 0.19^{*}$ & 0.000 \\
EP adipocyte volume, $p \mathrm{~L}$ & $350.80 \pm 22.42$ & $432.80 \pm 14.28^{*}$ & 0.014 \\
SC adipocyte volume, $p L$ & $257.90 \pm 28.31$ & $359.30 \pm 36.55^{*}$ & 0.041 \\
Glucose, $\mathrm{mg} / \mathrm{dL}$ & $132.91 \pm 4.73$ & $148.80 \pm 3.88^{*}$ & 0.035 \\
Insulin, $\mathrm{ng} / \mathrm{mL}$ & $0.53 \pm 0.04$ & $0.99 \pm 0.19^{*}$ & 0.022 \\
TNF- $\alpha, p g / \mathrm{mL}$ & $45.78 \pm 2.54$ & $59.05 \pm 3.94^{*}$ & 0.010 \\
Leptin, $\mathrm{ng} / \mathrm{mL}$ & $12.70 \pm 1.22$ & $25.40 \pm 2.78^{*}$ & 0.000 \\
Adiponectin, $n g / m L$ & $22.67 \pm 1.38$ & $16.38 \pm 1.28^{*}$ & 0.006 \\
\hline
\end{tabular}

SC, subcutaneous; EP, epididymal; RP, retroperitoneal

Values are mean \pm sem (control group, $n=10$; high-fat group, $n=7$ ) ${ }^{*} P<0.05$ vs. control diet group. 


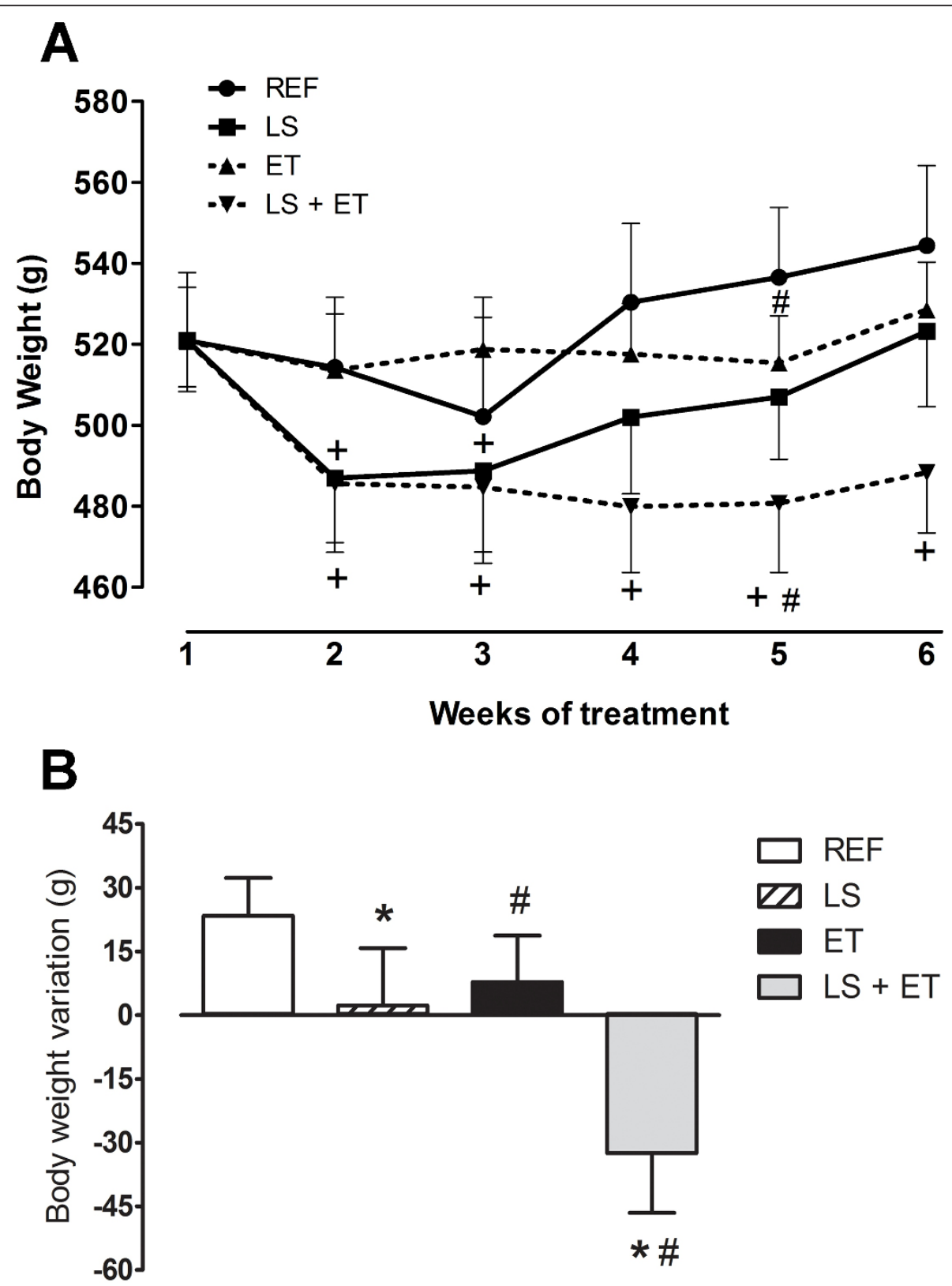

Figure 1 Effect of chronic exercise training and/or leucine supplementation on body weight changes. (A) Body weight. \#, effect of training in fifth week $(P=0.045)$; + vs week $1(P<0.002)$. (B) Total body weight variation (final - initial body weight). ${ }^{*}$, effect of diet $(P=0.028)$; \#, effect of training, $(P=0.006)$. Experimental groups: control diet $(R E F)$, diet supplemented with 5\% L-leucine $(L S)$, 6 weeks of endurance training (ET) plus standard diet, and diet supplemented with 5\% L-leucine plus endurance training (LS+ET). Values are mean \pm sem $(n=7-8$ rats per group).

weight loss that persisted throughout the experimental period $(P<0.001)$ (Figure $1 \mathrm{~A})$. When body weight variation was analyzed, both LS $(P=0.028)$ and ET $(P=$ $0.006)$ significantly reduced body weight gain. Nevertheless, analysis of treatment interaction showed similar responses in body weight variation for the two treatments (Figure 1B).
ET is more efficient than LS in reducing body adiposity in previously obese rats

ET reduced $(P=0.019)$ fat content in the carcass (Figure 2A). Furthermore, we found higher lean mass in trained animals compared to sedentary animals $(P=$ $0.019)$. However, there was no significant difference $(P>$ $0.05)$ in carcass protein or moisture content in trained 

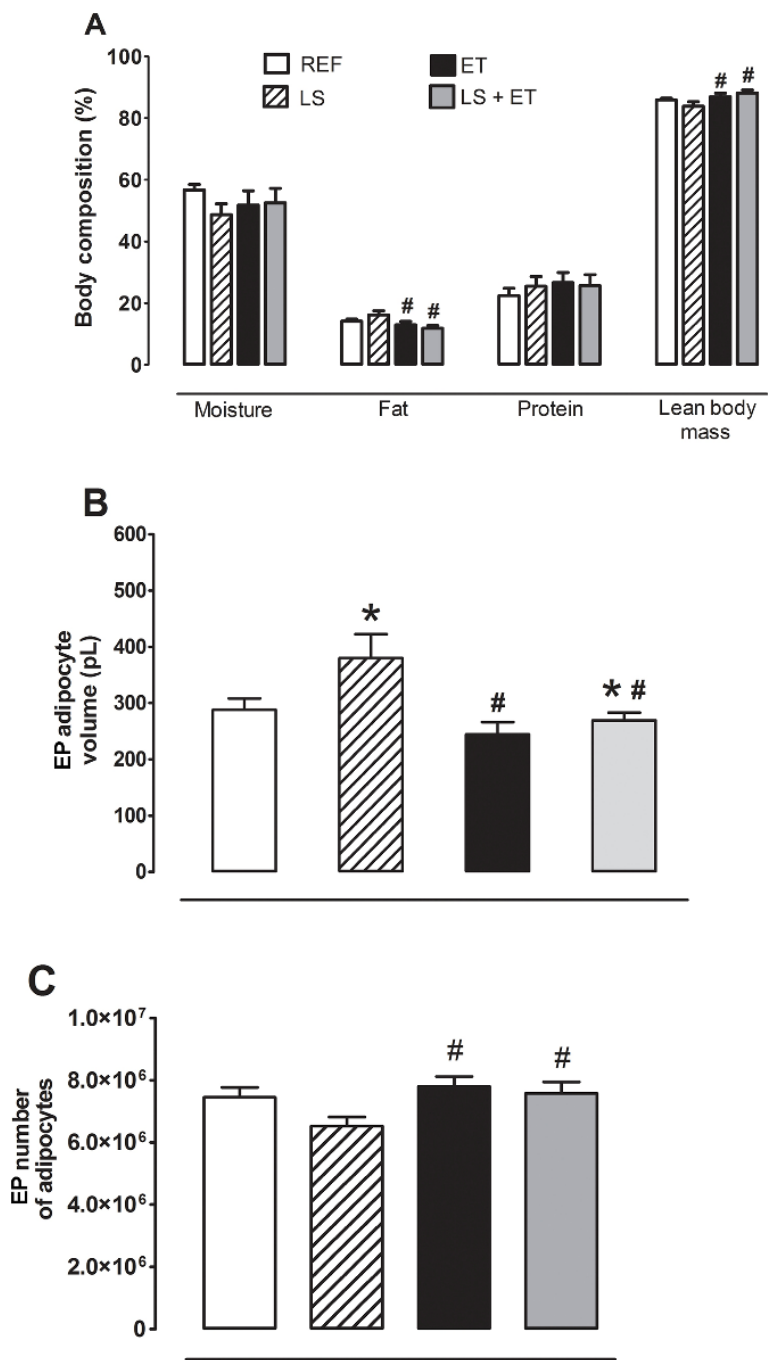

Figure 2 Chronic exercise is more efficient than leucine supplementation in reducing adiposity. (A) Body composition (\%). ${ }^{\#}$, effect of training $(P<0.019)$. (B) Epididymal adipocyte volume. ${ }^{*}$, effect of training $(P<0.005)$; ${ }^{*}$, effect of diet, $(P<0.020)$. (C) Epididymal adipocyte number \#, effect of training $(P<0.044)$. Experimental groups: control diet (REF), diet supplemented with $5 \%$ L-leucine (LS), 6 weeks of endurance training (ET) plus standard diet, and diet supplemented with $5 \%$ L-leucine plus endurance training $(\mathrm{LS}+\mathrm{ET})$. Values are mean $\pm \operatorname{sem}(n=7-8$ rats per group).

animals supplemented with leucine and no interaction between these variables was observed (Figure 2A).

EP fat pad weight was significantly $(P=0.033)$ lower in the trained group compared to the sedentary group (Table 3). Conversely, LS was less effective in reducing body fat as can be seen by the higher $(P=0.030)$ value obtained for the sum of fat pads (Table 3$)$. Both LS $(P=$ $0.020)$ and $\operatorname{ET}(P=0.005)$ induced distinct but significant effects on EP adipocyte volume (Figure 2B). In parallel, LS was less effective in reducing adipocyte expansion in the EP region, whereas ET significantly reduced EP adipocyte volume. Furthermore, ET increased the number of EP adipocytes $(P=0.044)$ (Figure $2 \mathrm{C}$ ).

\section{LS reduces serum total cholesterol levels in sedentary animals}

Both LS and ET did not induce significant differences in serum TG (Figure 3A) or HDL-cholesterol levels (Figure 3C). LS reduced serum total cholesterol concentration in sedentary animals $(P=0.042)$, but not in trained animals, since a significant difference was only observed for the interaction of the two factors $(P=0.018)$ (Figure 3B).

\section{ET is more efficient than LS in reducing insulin levels and HOMA index}

Serum glucose level was not significantly altered by diet or ET (Figure 3D). However, only ET significantly reduced $(P=0.022)$ serum insulin concentration (Figure $3 \mathrm{E})$. Regarding HOMA index, a strong trend $(P=0.059)$ in reducing insulin resistance status was seen in the trained animals when compared with sedentary groups, $\operatorname{REF}(0.29 \pm 0.05)$; LS $(0.32 \pm 0.06) ; \mathrm{ET}(0.21 \pm 0.05) ; \mathrm{LS}$ + ET $(0.20 \pm 0.03)$; diet $(P=0.872)$; training $(P=0.059)$, and interaction $(P=0.683)$.

\section{LS and ET differently affect the levels of adipokines}

Serum adiponectin level was significantly increased $(P=$ 0.021 for diet factor) after 6 weeks of LS (Figure 4A), but the same effect was not observed for ratio adiponectin/fat pad weight, REF $(3.81 \pm 0.43 \mathrm{ng} / \mathrm{mL} / 100 \mathrm{~g})$; $\mathrm{LS}$ $(5.56 \pm 0.75 \mathrm{ng} / \mathrm{mL} / 100 \mathrm{~g})$; ET $(3.66 \pm 0.39 \mathrm{ng} / \mathrm{mL} / 100$ g); LS+ET $(4.08 \pm 0.33 \mathrm{ng} / \mathrm{mL} / 100 \mathrm{~g})$; diet $(P=0.041)$; training $(P=0.121)$, and interaction $(P=0.202)$ and TNF- $\alpha$ (Figure 4B). ET was effective in significantly reducing $(P=0.044)$ TNF- $\alpha$ concentration when compared to the sedentary groups (Figure 4B), whereas serum leptin concentration was not significantly affected by either diet or ET (Figure 4C).

\section{Effect of LS and ET on the plasma concentration of amino acids}

Plasma leucine concentration was not significantly altered by diet $(P=0.952)$ or ET $(P=0.077)$ in overnight fasted rats. Moreover, significant differences were observed for some amino acids after overnight fasting, with the effects related to supplementation with leucine [glycine, $(P=0.008)$ and threonine, $(P=0.015)$ ], ET [proline, $(P=0.038)$ ] and we found some interactions [aspartic acid, $(P=0.018)$, glycine $(P=0.012)$, histidine $(P=0.031)$, threonine $(P=0.005)$, methionine $(P=$ $0.033)$ and taurine $(P=0.003)$ ] (Figure 5$)$.

\section{Discussion}

Previous studies have demonstrated improvement in the control of body weight or a reduction of body fat 
Table 3 Effect of chronic (6 weeks) physical training and/or leucine supplementation on fat pad weight

\begin{tabular}{llllllll}
\hline & & & & & \multicolumn{3}{l}{ 2-Way ANOVA P-values } \\
\cline { 5 - 8 } & REF & LS & ET & LS+ET & Diet & Training & Interaction \\
\hline SC, g/100 g b.w. & $2.72 \pm 0.20$ & $3.23 \pm 0.35$ & $2.59 \pm 0.21$ & $2.92 \pm 0.18$ & 0.110 & 0.397 & 0.717 \\
EP, g/100 g b.w. & $1.93 \pm 0.16$ & $2.45 \pm 0.29$ & $1.70 \pm 0.14$ & $1.82 \pm 0.07$ & 0.108 & $0.033^{*}$ & 0.301 \\
RP, g/100 g b.w. & $1.36 \pm 0.15$ & $1.78 \pm 0.23$ & $1.18 \pm 0.13$ & $1.41 \pm 0.09$ & 0.054 & 0.095 & 0.554 \\
$\sum, g / 100$ g b.W. & $6.01 \pm 0.45$ & $7.79 \pm 0.82$ & $5.47 \pm 0.45$ & $6.14 \pm 0.30$ & $0.030^{*}$ & 0.052 & 0.305 \\
\hline
\end{tabular}

(REF), control diet; (LS), diet supplemented with 5\% L-leucine; (ET), endurance training plus control diet; (LS+ET), diet supplemented with 5\% leucine plus endurance training.

SC, subcutaneous; EP, epididymal; RP, retroperitoneal; $\Sigma$, sum of fat pad weight.

Values are mean \pm sem ( $n=7-8$ rats per group).

*Effect of diet, $P<0.05$; \#effect of training, $P<0.05$.

content with leucine or branched-chain amino acid supplementation [5,8,30-32]. However, other studies have not observed these responses $[4,9,33]$. In some previous studies, animals were simultaneously exposed to a HFD and LS. Thus, these studies investigated the effect of leucine on the prevention of diet-induced obesity $[8,9]$. In our study, we first induced obesity, hyperglycemia and hyperinsulinemia in animals using a HFD. After establishment of diet-induced obesity, the animals received a control diet supplemented or not with leucine
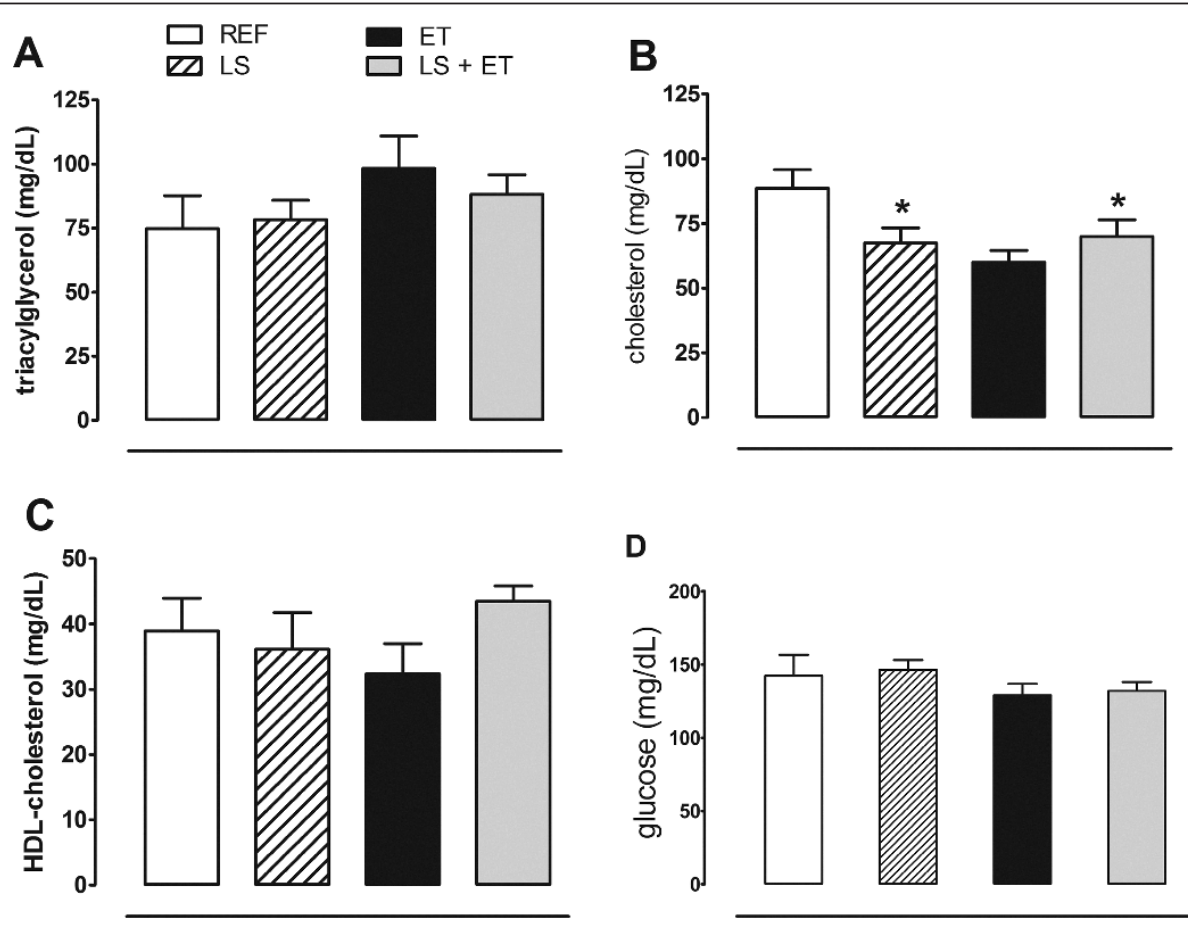

D
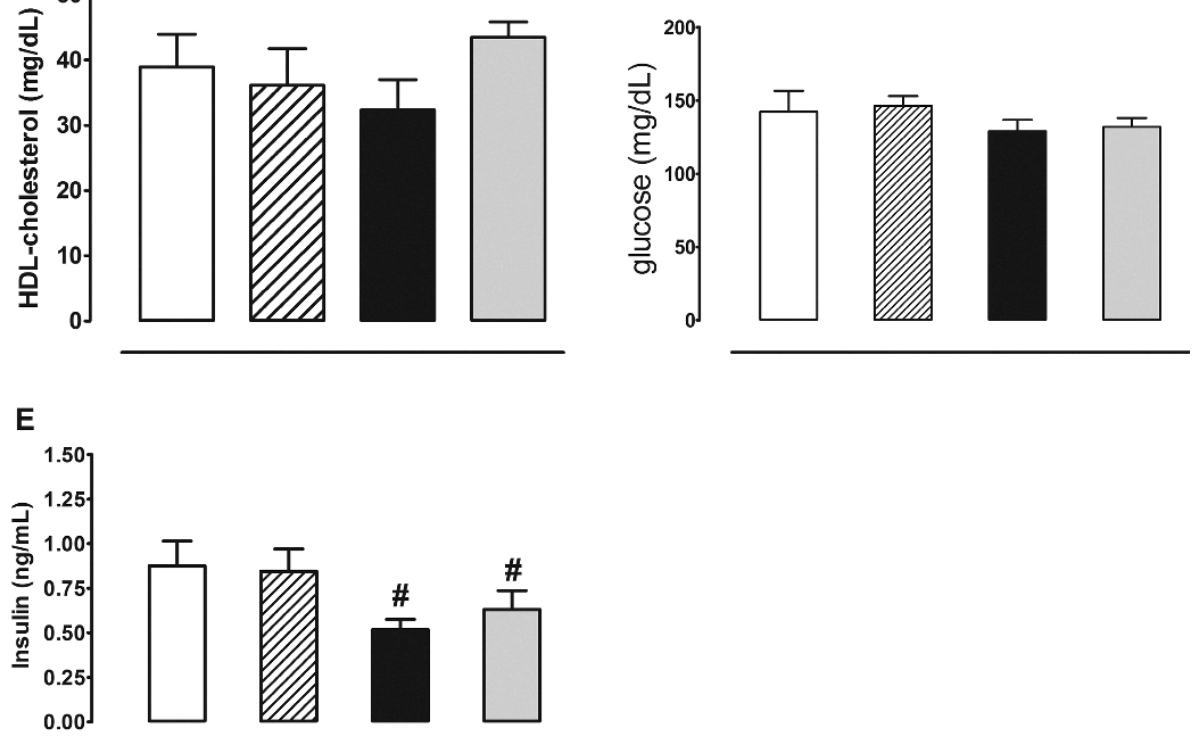

Figure 3 Leucine supplementation reduces serum total cholesterol levels in sedentary animals. (A) Serum concentration of triacylglycerol. (B) Serum concentration of cholesterol. *, effect of diet $(P<0.042)$; interaction of the two factors $(P=0.018)$. (C) Serum concentration of HDLcholesterol. (D) Serum concentration of glucose. (E) Serum concentration of Insulin. \#, effect of training $(P<0.022)$. Experimental groups: control diet (REF), diet supplemented with 5\% L-leucine (LS), 6 weeks of endurance training (ET) plus standard diet, and diet supplemented with 5\% Lleucine plus endurance training (LS+ET). Values are mean $\pm \operatorname{sem}(n=5-8$ rats per group). 


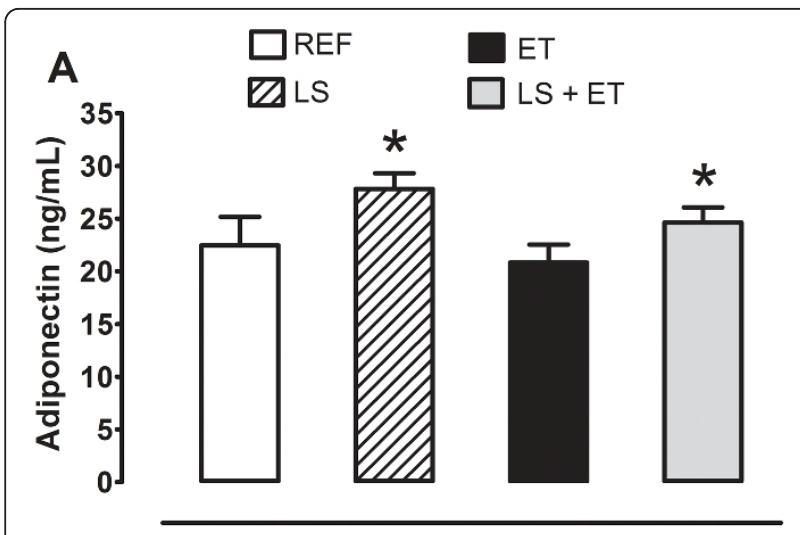

\section{B}

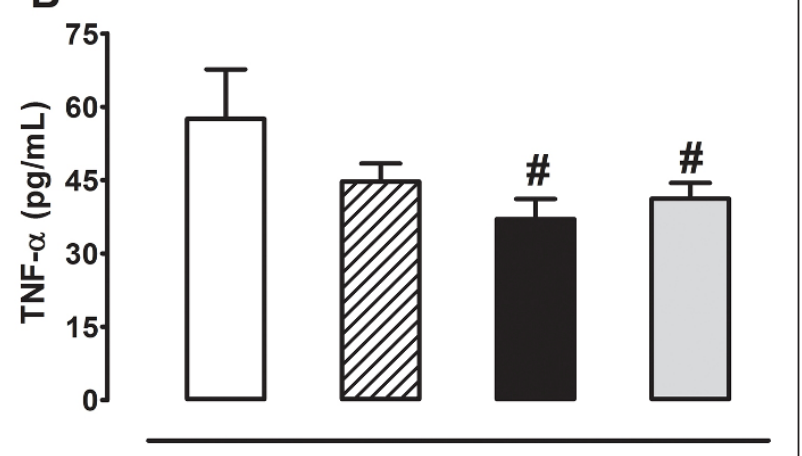

C

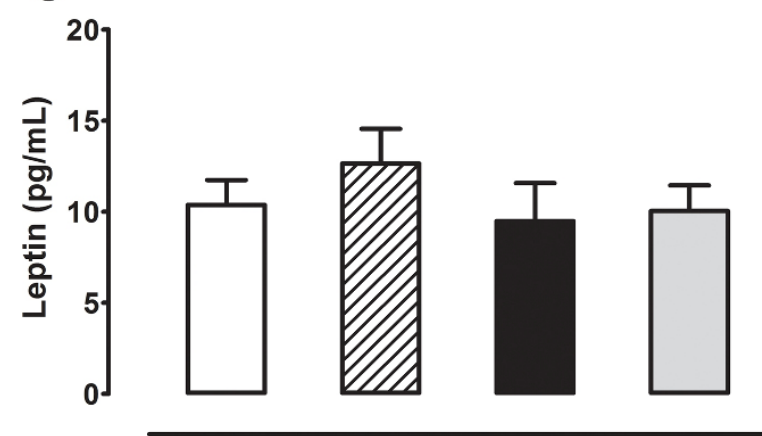

Figure 4 Leucine supplementation increases adiponectin levels. (A) Adiponectin. *, effect of diet $(P<0.021)$. (B) TNF- $\alpha$. ${ }^{*}$, effect of training $(P<0.044)$. (C) Leptin. Experimental groups: control diet (REF), diet supplemented with 5\% L-leucine (LS), 6 weeks of endurance training (ET) plus standard diet, and diet supplemented with $5 \%$ L-leucine plus endurance training (LS+ET). Values are mean $\pm \operatorname{sem}(n=6-8$ rats per group).

in order to study the potential of this amino acid in reverting the obesity, hyperglycemia and hyperinsulinemia phenotypes associated with long-term exposure to a HFD. Furthermore, we evaluated the effects of LS combined with ET, since physical exercise is a recognized strategy used to improve body weight control and insulin sensitivity. We found that chronic LS in this experimental paradigm did not alter food intake nor did it reduce fat pad mass or visceral adipocyte volume. We also showed that LS affected the endocrine function of adipocytes, increasing the circulating levels of adiponectin. Furthermore, we observed a beneficial effect of LS on the serum concentrations of total cholesterol. However, no changes in serum glucose, insulin, leptin, or TNF- $\alpha$ were seen. In comparison to LS alone, ET was more effective in attenuating the degree of adiposity, hyperinsulinemia and circulating levels of TNF- $\alpha$.

The model of obesity induced by HFD is widely accepted and has been used alone, or in combination with other strategies, as an animal model of excess body fat $[1,34,35]$. Our results validate the diet employed to induce obesity, confirming that rats chronically exposed to a HFD present elevated body fat with hypertrophy of SC and EP adipocytes, leptin resistance, abnormal glucose homeostasis and a proinflammatory state characterized by increased serum TNF- $\alpha$ and reduced serum adiponectin levels. Therefore, LS was evaluated in animals already showing indicators of metabolic syndrome $[1,34,35]$.

The present results indicate at transient effect of LS on body weight, since we observed a lower body weight at the beginning of supplementation. However, these animals recovered their weight later. Under these experimental conditions, leucine is unable to maintain its effect of reducing body weight for a prolonged period of supplementation. On the other hand, when supplementation is combined with ET body, weight loss is equally achieved and maintained during the treatment period.

With regard to adipose mass and adipocyte volume, we observed independent effects of LS and ET. In this case, whereas trained animals displayed reduced adiposity, leucine-treated animals had greater fat mass and fat cell volume. The present results regarding adiposity agree with the already known heterogeneity of fat pads. In this respect, LS favored a higher adiposity in the visceral region, irrespective of the fat pad assessed. Interestingly, ET affected the same fat pads, but in opposite way, favoring fat mass reduction.

This effect on adiposity observed in leucine-treated animals may be related to mammalian target of rapamycin (mTOR) activation. Although we did not directly assess mTOR activation in adipose tissue, our results are consistent with those obtained by Chakrabarti et al. [36]. These authors showed that activation of mTORC1 signaling in 3T3-L1 adipocytes transcriptionally inhibits the expression of adipose triglyceride lipase (ATGL) and hormone sensitive lipase (HSL), thus suppressing lipolysis of TG and diacylglycerol in adipose tissue. Moreover, these authors found that $\mathrm{mTORC} 1$ activation in adipocytes promotes de novo lipogenesis and TG accumulation. Accordingly, Polak et al. [37] observed that adipocytes from knockout animals for raptor, a regulatory protein associated with 


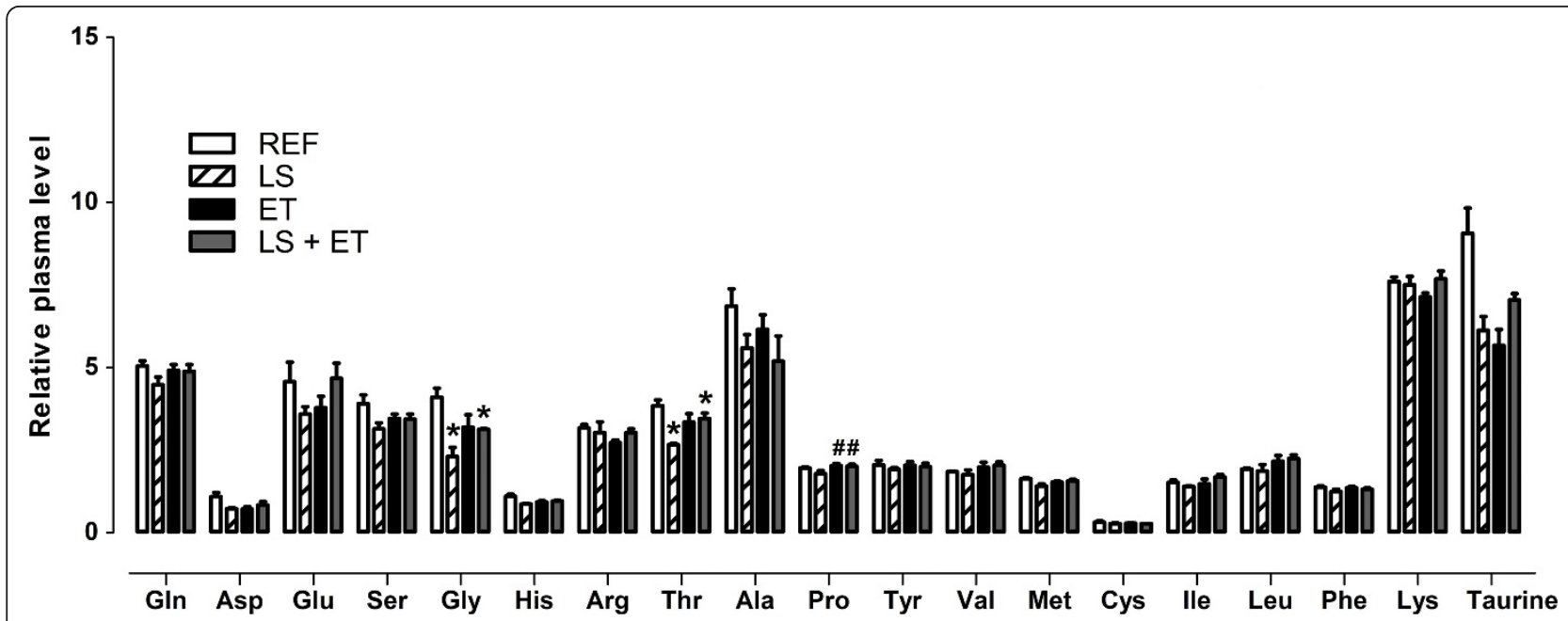

Figure 5 Effect of chronic exercise and/or leucine supplementation on circulating levels of amino acids. \#, effect of training $(P<0.05)$; ${ }^{*}$ interaction for amino acids glycine and threonine $(P<0.05)$. Experimental groups: control diet (REF), diet supplemented with $5 \% \mathrm{~L}-\mathrm{leucine}(\mathrm{LS}), 6$ weeks of endurance training (ET) plus standard diet, and diet supplemented with $5 \%$ L-leucine plus endurance training (LS+ET). Values are mean $\pm \operatorname{sem}(n=5-4$ rats per group).

rapamycin-sensitive mTOR and responsible for mTORC1 activity [38], had lower deposition of TG. In a recent study, Zeanandin et al. [23] demonstrated that 18-monthold rats showed hypertrophy and hyperplasia of adipose tissue after 6 months of LS. These effects were attributed to an increased phosphorylation of mTOR and increased mRNA expression of PPAR $\gamma$ in adipose tissue. This evidence suggests that mTOR signaling is an important intracellular pathway, directly promoting lipogenic processes in adipocytes.

Some studies have shown that LS reduces adiposity after dietary intervention, such as LS combined with food restriction [5,32]. Furthermore, several studies administered much lower doses of LS $(0.59 \%)[4,5,33]$ than that used in the present study (5\%). In addition, the initial metabolic condition of the animals in our study may have contributed to a potential lipogenic effect of LS. Thus, our results suggest that LS has few positive effects on the treatment of obesity, hyperglycemia and hyperinsulinemia when these conditions are already present. In addition, our in vivo data confirm in vitro studies [39] suggesting that, depending on the metabolic condition of the body, leucine exerts a lipogenic effect, possibly by activating mTORC1 in adipocytes [36,37].

Similarly, when insulin-resistant adipocytes were incubated in the presence of leucine and insulin, the recovery of lipogenesis and glucose uptake were observed [39]. From this perspective, these responses support our hypothesis that in the presence of insulin resistance leucine may retrieve the lipogenic effects of insulin, promoting body fat deposition, as proposed by Hinault et al. [39].
Based on our results regarding LS in initially hyperinsulinemic and hyperglycemic animals, no significant effects on serum glucose or insulin levels were observed. These effects agree with data from other studies in which LS did not affect body composition $[4,9]$. On the other hand, leucine-induced changes in hyperglycemia and hyperinsulinemia have been suggested to depend on changes in adiposity [8].

With regard to hormonal changes, we observed that the type of treatment had different effects on serum TNF- $\alpha$ (ET effect) and adiponectin (LS effect) levels. Whereas LS caused no changes in TNF- $\alpha$ concentration, ET reduced serum TNF- $\alpha$ levels. The concentrations of TNF- $\alpha$ are inversely proportional to the insulin response. Moreover, the lower serum TNF- $\alpha$ levels might be due to reduced adiposity in trained animals [40].

In vitro experiments showed that leucine promotes increased synthesis and secretion of adiponectin in 3T3-L1 adipocytes [12,13], and this effect may be mTOR-dependent [41]. Adiponectin is directly associated with improved glucose uptake by increasing insulin sensitivity [42]. However, the increased serum adiponectin level seen in our leucine-supplemented groups did not improve the glucose homeostasis of these animals. Studies suggest that increases in adiponectin concentration may be related to the increase of different isoforms of this adipokine [43], and some of these isoforms are not related to improvement of glucose metabolism or insulin sensitivity [43]. Thus, further studies are required to clarify which isoforms of adiponectin are stimulated by leucine and how this interferes with insulin sensitivity. 
Another effect of LS was its ability to reduce the serum concentration of total cholesterol, and this response did not depend on changes in body weight or fat mass. However, leucine was only able to reduce serum cholesterol levels in sedentary animals since the same was not seen in the trained group. This response related to LS agrees with the results of Zhang et al. [8] who observed that the reduction in cholesterol levels was largely independent of leucine-induced changes in adiposity. However, little is known about the molecular mechanisms activated by LS that are able to change cholesterol metabolism.

In conclusion, the present study provides further evidence indicating that the effects of leucine on body composition are highly dependent on the metabolic state of the animals at the beginning of LS. In this respect, several studies have shown no change or a reduction in fat mass when leucine is administered in situations that improve insulin sensitivity, such as food restriction [5] or combined with physical exercise (present study), or simultaneously with a HFD $[8,9,44]$. On the other hand, a lipogenic effect of leucine treatment is observed when the amino acid is administered to cells previously exposed to situations that induce insulin resistance [39]. Therefore, our findings support a potential lipogenic role of leucine under conditions of preexisting hyperglycemia and hyperinsulinemia as suggested in other studies. The results also showed that ET combined with LS had no additional beneficial effects other than those seen with either treatment alone. Finally, although leucine had no beneficial effects on insulin sensitivity, LS was effective in decreasing the circulating levels of cholesterol and in increasing serum adiponectin concentration.

\section{List of Abbreviations used}

ATGL: adipose triglyceride lipase; CD: control diet; ET: endurance training; ELISA: enzyme-linked immunosorbent assay; EP: epididymal; HDL-c: highdensity lipoprotein-cholesterol; HFD: high-fat diet; HSL: hormone sensitive lipase; IL-6: interleukin-6; LS: leucine supplementation; mTOR: mammalian target of rapamycin; REF: reference; RP: retroperitoneal; SC: subcutaneous; TG: triacylglycerol; TNF-a: tumor necrosis factor-alpha.

\section{Acknowledgements \\ The authors thank Ajinomoto do Brazil for the donation of the purified amino acid L-leucine, Conselho Nacional de Desenvolvimento Científico e Tecnológico (CNPq), and Coordenação de Aperfeiçoamento de Pessoal de Nível Superior (CAPES). This investigation was supported by grants $07 / 51964$ 9 (Torres-Leal FL), 07/56230-3, 07/59291-3 (Tirapegui J), and 08/58428-8 (Capitani MD) from the Fundação de Amparo a Pesquisa do Estado de São Paulo (FAPESP), Brazil. We also acknowledge contributions from the following laboratory member: Ivanir Santana Pires. All authors read and approved the final manuscript.}

\section{Author details}

${ }^{1}$ Department of Food Science and Experimental Nutrition, Faculty of Pharmaceutical Sciences, University of São Paulo, São Paulo, Brazil. ${ }^{2}$ Heart Institute (InCor), University of Sao Paulo Medical School, Sao Paulo, Brazil. ${ }^{3}$ Department of Nutrition, School of Public Health, São Paulo University, Sao
Paulo, Brazil. ${ }^{4}$ Department of Internal Medicine, Division of Hypothalamic Research, University of Texas Southwestern Medical Center, Dallas, TX 75390 - USA.

\section{Authors' contributions}

For experimental design: FLTL, JDJr, MHFA, MMR, JT; for data collection: FLTL, MHFA, GFRT, MDC, DV, LCP, EMMN; for data analysis: FLTL, JDJr, MHFA, $M M R$, JT; for drafting the manuscript: FLTL, JDJr, MHFA, MMR, JT. All authors read and approved the final manuscript.

\section{Competing interests}

The authors declare that they have no competing interests.

Received: 3 May 2011 Accepted: 7 September 2011

Published: 7 September 2011

\section{References}

1. Arcari DP, Bartchewsky W, dos Santos TW, Oliveira KA, Funck A, Pedrazzoli J, de Souza MF, Saad MJ, Bastos DH, Gambero A, et al: Antiobesity effects of yerba mate extract (llex paraguariensis) in high-fat diet-induced obese mice. Obesity (Silver Spring) 2009, 17:2127-2133.

2. Prada PO, Hirabara SM, de Souza CT, Schenka AA, Zecchin HG, Vassallo J, Velloso LA, Carneiro E, Carvalheira JB, Curi R, Saad MJ: L-glutamine supplementation induces insulin resistance in adipose tissue and improves insulin signalling in liver and muscle of rats with diet-induced obesity. Diabetologia 2007, 50:1949-1959.

3. Hotamisligil GS: Inflammation and metabolic disorders. Nature 2006, 444:860-867.

4. Donato J, Pedrosa RG, de Araujo JA, Pires IS, Tirapegui J: Effects of leucine and phenylalanine supplementation during intermittent periods of food restriction and refeeding in adult rats. Life Sci 2007, 81:31-39.

5. Donato J, Pedrosa RG, Cruzat VF, Pires IS, Tirapegui J: Effects of leucine supplementation on the body composition and protein status of rats submitted to food restriction. Nutrition 2006, 22:520-527.

6. Halton $\mathrm{TL}, \mathrm{Hu}$ FB: The effects of high protein diets on thermogenesis, satiety and weight loss: a critical review. J Am Coll Nutr 2004, 23:373-385.

7. Layman DK: The role of leucine in weight loss diets and glucose homeostasis. J Nutr 2003, 133:261S-267S.

8. Zhang Y, Guo K, LeBlanc RE, Loh D, Schwartz GJ, Yu YH: Increasing dietary leucine intake reduces diet-induced obesity and improves glucose and cholesterol metabolism in mice via multimechanisms. Diabetes 2007, 56:1647-1654.

9. Nairizi A, She P, Vary TC, Lynch CJ: Leucine supplementation of drinking water does not alter susceptibility to diet-induced obesity in mice. J Nutr 2009, 139:715-719.

10. Cota D, Proulx K, Smith KA, Kozma SC, Thomas G, Woods SC, Seeley RJ: Hypothalamic mTOR signaling regulates food intake. Science 2006, 312:927-930.

11. Lynch CJ, Gern B, Lloyd C, Hutson SM, Eicher R, Vary TC: Leucine in food mediates some of the postprandial rise in plasma leptin concentrations. Am J Physiol Endocrinol Metab 2006, 291:E621-630.

12. Blumer RM, van Roomen CP, Meijer AJ, Houben-Weerts JH, Sauerwein HP, Dubbelhuis PF: Regulation of adiponectin secretion by insulin and amino acids in 3T3-L1 adipocytes. Metabolism 2008, 57:1655-1662.

13. Sun $X$, Zemel MB: Leucine and calcium regulate fat metabolism and energy partitioning in murine adipocytes and muscle cells. Lipids 2007, 42:297-305.

14. Jakicic JM: The effect of physical activity on body weight. Obesity (Silver Spring) 2009, 17 Suppl 3:S34-38.

15. Donnelly JE, Blair SN, Jakicic JM, Manore MM, Rankin JW, Smith BK: American College of Sports Medicine Position Stand. Appropriate physical activity intervention strategies for weight loss and prevention of weight regain for adults. Med Sci Sports Exerc 2009, 41:459-471.

16. Sloan RP, Shapiro PA, Demeersman RE, McKinley PS, Tracey KJ, Slavov I, Fang Y, Flood PD: Aerobic exercise attenuates inducible TNF production in humans. J Appl Physiol 2007, 103:1007-1011.

17. Pischon T, Hankinson SE, Hotamisligil GS, Rifai N, Rimm EB: Leisure-time physical activity and reduced plasma levels of obesity-related inflammatory markers. Obes Res 2003, 11:1055-1064.

18. Lim S, Choi SH, Jeong IK, Kim JH, Moon MK, Park KS, Lee HK, Kim YB, Jang HC: Insulin-sensitizing effects of exercise on adiponectin and 
retinol-binding protein- 4 concentrations in young and middle-aged women. J Clin Endocrinol Metab 2008, 93:2263-2268.

19. Kondo T, Kobayashi I, Murakami M: Effect of exercise on circulating adipokine levels in obese young women. Endocr J 2006, 53:189-195

20. Layman DK, Evans E, Baum Jl, Seyler J, Erickson DJ, Boileau RA: Dietary protein and exercise have additive effects on body composition during weight loss in adult women. J Nutr 2005, 135:1903-1910.

21. Reeves PG, Nielsen FH, Fahey GC Jr: AIN-93 purified diets for laboratory rodents: final report of the American Institute of Nutrition ad hoc writing committee on the reformulation of the AIN-76A rodent diet. J Nutr 1993, 123:1939-1951.

22. Tsubuku S, Hatayama K, Katsumata T, Nishimura N, Mawatari K, Smriga M, Kimura T: Thirteen-week oral toxicity study of branched-chain amino acids in rats. Int J Toxicol 2004, 23:119-126.

23. Zeanandin G, Balage M, Schneider SM, Dupont J, Hebuterne X, MotheSatney I, Dardevet D: Differential effect of long-term leucine supplementation on skeletal muscle and adipose tissue in old rats: an insulin signaling pathway approach. Age (Dordr) 2011

24. de Araujo JA, Falavigna G, Rogero MM, Pires IS, Pedrosa RG, Castro IA, Donato J, Tirapegui J: Effect of chronic supplementation with branchedchain amino acids on the performance and hepatic and muscle glycogen content in trained rats. Life Sci 2006, 79:1343-1348.

25. Rogero MM, Tirapegui J, Pedrosa RG, Castro IA, Pires IS: Effect of alanylglutamine supplementation on plasma and tissue glutamine concentrations in rats submitted to exhaustive exercise. Nutrition 2006, 22:564-571.

26. Rodbell M: Metabolism of Isolated Fat Cells. I. Effects of Hormones on Glucose Metabolism and Lipolysis. J Biol Chem 1964, 239:375-380.

27. Fine JB, DiGirolamo M: A simple method to predict cellular density in adipocyte metabolic incubations. Int J Obes Relat Metab Disord 1997, 21:764-768.

28. White JA, Hart RJ, Fry JC: An evaluation of the Waters Pico-Tag system for the amino-acid analysis of food materials. J Automat Chem 1986, 8:170-177

29. Hagen SR, Frost B, Augustin J: Precolumn phenylisothiocyanate derivatization and liquid chromatography of amino acids in food. J Assoc Off Anal Chem 1989, 72:912-916.

30. Layman DK, Boileau RA, Erickson DJ, Painter JE, Shive H, Sather C, Christou DD: A reduced ratio of dietary carbohydrate to protein improves body composition and blood lipid profiles during weight loss in adult women. J Nutr 2003, 133:411-417.

31. Layman DK, Walker DA: Potential importance of leucine in treatment of obesity and the metabolic syndrome. J Nutr 2006, 136:319S-323S.

32. Mourier A, Bigard AX, de Kerviler E, Roger B, Legrand H, Guezennec CY: Combined effects of caloric restriction and branched-chain amino acid supplementation on body composition and exercise performance in elite wrestlers. Int I Sports Med 1997, 18:47-55

33. Pedrosa RG, Donato J, Pires IS, Tirapegui J: Leucine supplementation favors liver protein status but does not reduce body fat in rats during 1 week of food restriction. Appl Physiol Nutr Metab 2010, 35:180-183.

34. Carvalho-Filho MA, Ropelle ER, Pauli RJ, Cintra DE, Tsukumo DM, Silveira LR, Curi R, Carvalheira JB, Velloso LA, Saad MJ: Aspirin attenuates insulin resistance in muscle of diet-induced obese rats by inhibiting inducible nitric oxide synthase production and S-nitrosylation of IRbeta/IRS-1 and Akt. Diabetologia 2009, 52:2425-2434.

35. Milanski M, Degasperi G, Coope A, Morari J, Denis R, Cintra DE, Tsukumo DM, Anhe G, Amaral ME, Takahashi HK, et al: Saturated fatty acids produce an inflammatory response predominantly through the activation of TLR4 signaling in hypothalamus: implications for the pathogenesis of obesity. J Neurosci 2009, 29:359-370.

36. Chakrabarti P, English T, Shi J, Smas CM, Kandror KV: Mammalian target of rapamycin complex 1 suppresses lipolysis, stimulates lipogenesis, and promotes fat storage. Diabetes 2010, 59:775-781.

37. Polak P, Cybulski N, Feige JN, Auwerx J, Ruegg MA, Hall MN: Adiposespecific knockout of raptor results in lean mice with enhanced mitochondrial respiration. Cell Metab 2008, 8:399-410.

38. Oshiro N, Yoshino K, Hidayat S, Tokunaga C, Hara K, Eguchi S, Avruch J, Yonezawa K: Dissociation of raptor from mTOR is a mechanism of rapamycin-induced inhibition of mTOR function. Genes Cells 2004, 9:359-366.
39. Hinault C, Mothe-Satney I, Gautier N, Lawrence JC, Van Obberghen E: Amino acids and leucine allow insulin activation of the PKB/mTOR pathway in normal adipocytes treated with wortmannin and in adipocytes from db/db mice. FASEB J 2004, 18:1894-1896.

40. Bradley RL, Jeon JY, Liu FF, Maratos-Flier E: Voluntary exercise improves insulin sensitivity and adipose tissue inflammation in diet-induced obese mice. Am J Physiol Endocrinol Metab 2008, 295:E586-594.

41. Zhang HH, Huang J, Duvel K, Boback B, Wu S, Squillace RM, Wu CL, Manning BD: Insulin stimulates adipogenesis through the Akt-TSC2mTORC1 pathway. PLoS One 2009, 4:e6189.

42. Torres-Leal FL, Fonseca-Alaniz MH, Rogero MM, Tirapegui J: The role of inflamed adipose tissue in the insulin resistance. Cell Biochem Funct 2010, 28:623-631.

43. Pajvani UB, Du X, Combs TP, Berg AH, Rajala MW, Schulthess T, Engel J, Brownlee M, Scherer PE: Structure-function studies of the adipocytesecreted hormone Acrp30/adiponectin. Implications for metabolic regulation and bioactivity. J Biol Chem 2003, 278:9073-9085.

44. Ropelle ER, Pauli JR, Fernandes MF, Rocco SA, Marin RM, Morari J, Souza KK, Dias MM, Gomes-Marcondes MC, Gontijo JA, et al: A central role for neuronal AMP-activated protein kinase (AMPK) and mammalian target of rapamycin (mTOR) in high-protein diet-induced weight loss. Diabetes 2008, 57:594-605.

doi:10.1186/1743-7075-8-62

Cite this article as: Torres-Leal et al:: Leucine supplementation improves adiponectin and total cholesterol concentrations despite the lack of changes in adiposity or glucose homeostasis in rats previously exposed to a high-fat diet. Nutrition \& Metabolism 2011 8:62.

\section{Submit your next manuscript to BioMed Central and take full advantage of:}

- Convenient online submission

- Thorough peer review

- No space constraints or color figure charges

- Immediate publication on acceptance

- Inclusion in PubMed, CAS, Scopus and Google Scholar

- Research which is freely available for redistribution

Submit your manuscript at www.biomedcentral.com/submit
Biomed Central 